\title{
Middle Ear Cleft Pressure Variations: Role of the Eustachian Tube
}

Disturbances of middle ear gas exchange are responsible for highly prevalent symptoms and diseases. Symptoms can range from a discrete sensation of pressure in the ear to hearing impairment with socio-economic consequences.

Diseases range from tympanic membrane retraction, acute otitis media and serosa to chronic otitis media. Dysfunction of middle ear ventilation may also influence inner ear function leading to instability and vertigo. Sometimes it may also jeopardise air travelling and leisure activities such as diving.

The development of tympanometry into a routine noninvasive clinical tool greatly influenced and enhanced the insight into an important aspect of middle ear ventilation, i.e. middle ear pressure. The key function of the Eustachian tube (ET) in middle ear ventilation became apparent. Tympanometry also allowed functional evaluation of the ET by performing active movements like Valsalva and Toynbee manoeuvres and swallowing. Although sometimes criticised as unphysiological, these tests contributed to the understanding of the pathological consequences of dysfunction.

Quite soon it became clear that the ET not only plays a role in the ventilation of the middle ear, but also exerts a protective function for the middle ear against nasal pharyngeal flora and is involved in the clearance of middle ear secretions.

Subsequently the ventilatory key role of the ET was challenged by the observation that gas exchanges occur- ring during ET opening by swallowing in physiological conditions accounted only for a small fraction of middle ear ventilation. It was also shown that the middle ear cleft is not filled with air but with a gas mixture of a different composition. Gas exchanges at the level of the middle ear contribute mostly to this gas composition. Furthermore the role of the ET in pathology was questioned due to the lack of mechanical obstruction as assessed by ET endoscopy. Also pressure-regulating mechanisms of the mastoid and of tympanic membrane displacement were taken into account.

In view of the initial mechanical function of the ET, transtympanic ventilating tubes seemed to offer the ideal solution. The subsequent controversy on the ET and the lack of properly designed studies at that time caused a decrease in indications for transtympanic ventilating tubes.

Other proposed surgical interventions on the protympanum, ET and torus tubarius and placement of endoluminal stents have never become valid alternative therapies for ET dysfunction, neither did any medical therapy including steroids.

The current concept that the middle ear mucosa, the ET and the rhinopharynx have to be considered as a functional organ system offers better prospects for a more physiological approach taking into account the different mechanisms involved. Due to new methodologies and technical developments, more appropriate insights are emerging. Examples are long-term comparative outcome 
studies of different surgical procedures, e.g. demonstrating the disease-modifying role of adenoidectomy, or the application of immunobiology demonstrating the innate and adaptive immunological processes with their inductor and effect of sites at the level of the middle ear mucosa, ET and rhinopharynx.

Also molecular biology and for instance regulation of gene expression on mucins will find their application in this field.

In the current issue, four papers highlight the state of the art of some aspects of the regulation of middle ear gas exchange. The papers of Decraemer and Dirckx assess objectively the contribution of the different parts of the tympanic membrane in middle ear pressure regulation and they challenge former statements. The paper of Ars focuses particularly on the relation between function and anatomy of the middle ear mucosa and highlights in particular the aspect of gas exchange. At last the paper of Mulder et al. investigates, from an animal experimental view, how ET anatomy develops in order to cope with its function of ventilation and clearance.

We anticipate that the new technologies and approaches will introduce a new wave of interest in the ET, which will change the current stagnation in the therapeutic field of pathological middle ear gas exchange.

$P$. Van de Heyning

B. Ars

J. Claes

H. Valcke

Antwerp 